\section{Библиографрический список}

1. ГОСТ 27.002-89. Надежность в технике. Основные понятия. Термины и определения. М., 1990.

2. Новожилов Г. В., Неймарк М. С., Цесарский Л. Г. Безопасность полета самолета : концепция и технология. М. : Изд-во МАИ, 2007. 196 с.

3. Резчиков А. Ф., Твердохлебов В. А. Причинно-следственные модели производственных систем. Саратов : Научная книга, 2008.

4. Клюев В. В., Резчиков А. Ф., Богомолов А. С.,
Уков Д. А., Филимонюк Л. Ю. Системный подход к задаче оценки остаточного ресурса человекомашинных систем // Контроль. Диагностика. 2011. № 8. С. 9-13.

5. Клюев В. В., Резчиков А. Ф., Богомолов А. С., Кошевая Е. М., Уков Д. А. Причинно-следственный подход к управлению ресурсом печи обжига при производстве цемента // Контроль. Диагностика. 2012. № 7. C. $30-36$.

\title{
Integrated Resource Control of Complex Man-Machine Systems
}

\section{A. S. Bogomolov}

\begin{abstract}
Saratov State University, Russia, 410012, Saratov, Astrakhanskaya st., 83, alexbogomolov@ya.ru
Investigate the nature of emergencies and disasters in complex human-machine systems. In order to assess and predict the complex emergency proposed to consider the resource system as a vector comprising human resources and computer resources. Prediction of accidents are encouraged to check with the logical security conditions applicable to heterogeneous system resources in the complex
\end{abstract}

Key words: accidents, security, disaster, remaining life, man-machine systems, the human factor.

\section{References}

1. State Standard 27.002-89. Industrial product dependability. General concepts. Terms and definitions. Moscow, Standartinform, 1990, 24 p. (in Russian).

2. Novojilov G. V., Neymark M. S., Tsesarskiy L. G. Bezopasnost' poleta samoleta. Koncepcija i tehnologija [Providing of the flight safety of an airplane. The concept and technique]. Moscow, MAI, 2007, 196 p. (in Russian). 3. Rezchikov A. F., Tverdohlebov V. A. Prichinno-sledstvennye modeli proizvodstvennyh sistem [Cause-effect models of production systems]. Saratov, Nauchnaja kniga, 2008 (in Russian).

УДК 681.3.06, 681.322
4. Klyuev V. V, Rezchikov A. F., Bogomolov A. S., Ukov D. A., Filimonyuk L. Yu. System approach to a problem of appraisal of man-machine systems' remaining life. Control. Diagnostika, 2011, no. 8, pp. 9-13 (in Russian).

5. Kluev V. V., Rezchikov A. F., Bogomolov A. S., Koshevaya E. M., Ukov D. A. Cause-conditional approach to resource management of furnace in cement production. Control. Diagnostika, 2012, no. 7, pp. 30-36 (in Russian).

\section{ИССЛЕДОВАНИЕ ЭФФЕКТИВНОСТИ ПОИСКА ГЛОБАЛЬНОГО ЭКСТРЕМУМА МЕТОДОМ ИМИТАЦИИ ОТЖИГА, РАСПАРАЛЛЕЛЕННОГО РАЗЛИЧНЫМИ СПОСОБАМИ}

\footnotetext{
А. В. Высоцкий ${ }^{1}$, А. С. Тараканов ${ }^{1}$, К. И. Шоломов ${ }^{1}$, Н. Е. Тимофеева ${ }^{2}$, А. А. Ерофтиев ${ }^{3}$

${ }^{1}$ Студент касредры дискретной математики и инсрормационных технологий, Саратовский государственный университет им. Н. Г. Чернышевского, alex_v2008@mail.ru, sholomov@mail.ru, tarakanov.alexey@gmail.com

${ }^{2}$ Ассистент кафредры дискретной математики и инсрормационных технологий, Саратовский государственный университет им. Н. Г. Чернышевского, timofeevane@info.sgu.ru

${ }^{3}$ Аспирант кафредры математического обеспечения вычислительных комплексов и систем, Саратовский государственный университет им. Н. Г. Чернышевского, eroftiev.andrey@gmail.com

В данной статье представлены результаты создания параллельной вычислительной системы и проверки ее возможностей при решении учебных и научных задач. Рассмотрены и реализованы три варианта распараллеливания алгоритма оптимизации многоэкстремальной целевой фрункции двух переменных с явными ограничениями, основанного на стохастическом методе имитации отжига. Исследована зависимость надёжности и производительности параллельных версий алгоритма от их параметров и количества узлов параллельной вычислительной системы. Показано, что предложенные параллельные варианты алгоритма имитации отжига позволяют успешно находить глобальный минимум многоэкстремальной целевой функции.
}

Ключевые слова: глобальная оптимизация, алгоритм имитации отжига, многоэкстремальная целевая фрункция, явные ограничения, параллельные вычислительные системы. 


\section{ВВЕДЕНИЕ}

Современные задачи науки и техники, такие как моделирование климата, генная инженерия, проектирование интегральных схем, анализ загрязнения окружающей среды, создание лекарственных препаратов, наноматериалов и др., требуют для своего решения ЭВМ с огромной вычислительной производительностью. Единичные рабочие станции уже не способны в короткие сроки решать вышеперечисленные задачи. Здесь и возникает необходимость использования параллельных вычислений, когда над общей задачей работают десятки, сотни и даже тысячи рабочих станций.

Цель данной работы - реализация и проведение сравнительного анализа методов распараллеливания алгоритма нахождения глобального минимума многоэкстремальной целевой функции многих переменных с явными ограничениями (ограничениями типа равенств), основанного на стохастическом методе имитации отжига.

При этом решались следующие задачи:

- поиск эффективных способов распараллеливания алгоритма, с точки зрения минимизации времени и повышения надежности вычислений;

- программная реализация поиска глобального экстремума методом имитации отжига, распараллеленного различными способами;

- проведение численного эксперимента для оценки эффективности способов распараллеливания;

- анализ результатов численного эксперимента.

\section{1. ПОИСК ГЛОБАЛЬНОГО МИНИМУМА МЕТОДОМ ИМИТАЦИИ ОТЖИГА ПРИ НАЛИЧИИ ЯВНЫХ ОГРАНИЧЕНИЙ}

Метод отжига - это техника оптимизации, использующая упорядоченный случайный поиск на основе аналогии с процессом образования в веществе кристаллической структуры с минимальной энергией при охлаждении.

Большим преимуществом метода отжига является возможность избежать так называемых «ловушек» в локальных минимумах оптимизируемой функции за счет принятия изменений, временно ухудшающих результат, что отражает нагрев расплава для предотвращения его быстрого остывания при протекании соответствующего физического процесса. Еще одним преимуществом является то, что даже в условиях нехватки вычислительных ресурсов для нахождения глобального минимума метод отжига, как правило, выдает неплохое решение - один из локальных минимумов [1].

Конкретная схема отжига задается следующими параметрами [2]:

- законом изменения температуры $T(k)$, где $k$ - номер шага;

- порождающим семейством вероятностных распределений $\zeta(x, T)$;

- функцией вероятности принятия нового состояния $h(\Delta E, T)$.

Таким образом, алгоритм имитации отжига можно записать в следующем виде:

1) случайным образом выбирается начальная точка $x=x_{0}, x_{0} \in \Omega$. Текущее значение энергии $E$ устанавливается в значение $f\left(x_{0}\right)$.

2) $k$-я итерация алгоритма:

a) сравнить энергию системы $E$ в состоянии $x$ с найденным на настоящий момент глобальным минимумом. Если $E<\min , E=f(x)$, то изменить значение глобального минимума;

b) сгенерировать новую точку $x^{\prime}=G(x, T(k))$;

c) вычислить значение функции в ней: $E^{\prime}=f\left(x^{\prime}\right)$;

d) сгенерировать случайное число $\alpha$ из интервала $[0 ; 1]$;

е) если $\alpha<h\left(E^{\prime}-E, T(k)\right)$, то установить $x=x^{\prime}, E=E^{\prime}$, и перейти к следующей итерации. Иначе повторить шаг $b$, пока не будет найдена подходящая точка $x^{\prime}$.

Исторически первой схемой метода имитации отжига является схема больцмановского отжига. В ней изменение температуры задаётся в виде

$$
T(k)=\frac{T_{0}}{\ln (1+k)}, \quad k>0 .
$$

Порождающее семейство вероятностных распределений $\zeta(x, T)$ выбирается как семейство нормальных распределений с математическим ожиданием $x$ и дисперсией $T$ и соответственно задаётся плотностью

$$
g\left(x^{\prime} ; x, T\right)=(2 \pi T)^{-n / 2} \cdot \exp \left(-\left|x^{\prime}-x\right|^{2} /(2 T)\right),
$$

где $n-$ размерность метрического пространства состояний. 
Для данной схемы доказано, что при достаточно больших $T_{0}$ и количестве шагов $k$ гарантируется нахождение глобального минимума.

Недостатком схемы больцмановского отжига является очень медленное уменьшение температуры T. Решение этой проблемы возможно путём замены закона изменения температуры, например, на следующий $T(k)=r \cdot T(k-1)$, где температурный коэффициент $r$ выбирается, как правило, в пределах $0.7 \div 0.99$.

Такая схема имитации отжига называется тушением. Она очень быстро сходится, что позволяет экономить вычислительные ресурсы. При этом не гарантируется нахождения глобального минимума, но, как правило, быстро находится близкое решение, а на практике и сам минимум.

\section{2. ПАРАЛЛЕЛЬНЫЕ ВАРИАНТЫ МЕТОДА ИМИТАЦИИ ОТЖИГА}

Появление и развитие параллельных вычислительных систем привело к развитию нескольких подходов к распараллеливанию метода имитации отжига.

Существуют различные подходы к реализации алгоритма имитации отжига на параллельных вычислительных системах, рассмотрим 3 из них:

1) параллельный запуск алгоритма имитации отжига (мультистарт);

2) параллельный запуск алгоритма имитации отжига с обменом результатами (кластерный алгоритм);

3) разбиение пространства решений на области [3].

Рассмотрим их подробнее.

\section{1. Параллельный запуск алгоритма имитации отжига}

Данная адаптация метода имитации отжига предполагает вычисление глобального минимума ЦФ одновременно на нескольких машинах (вычислительных узлах) с последующим выбором лучшего решения на выделенной машине (управляющий узел).

В выполняемой на нескольких вычислительных узлах при одинаковых начальных условиях осуществляется поиск минимума ЦФ методом имитации отжига по схеме больцмановского тушения.

Затем результаты, полученные в вычислительных узлах, передаются в управляющий узел, в котором осуществляется выбор решения с наименьшим значением ЦФ, что должно обеспечивать глобальность найденного минимума.

Реализованный таким образом на параллельной вычислительной системе алгоритм оптимизации методом имитации отжига должен обеспечивать надёжный поиск глобального минимума целевой функции за счёт многократного повторения поиска в заданной явными ограничениями области.

При этом, так как многократный процесс поиска минимума осуществляется одновременно на соответствующем числе машин, то затраченное на выполнения всего параллельного алгоритма, блок-схема которого изображена на рис. 1, время приблизительно равно времени выполнения одного процесса и практически не зависит от числа процессов. Соответственно при использовании клиентских машин можно уменьшить временные затраты при поиске глобального минимума примерно в $p$ раз [2].

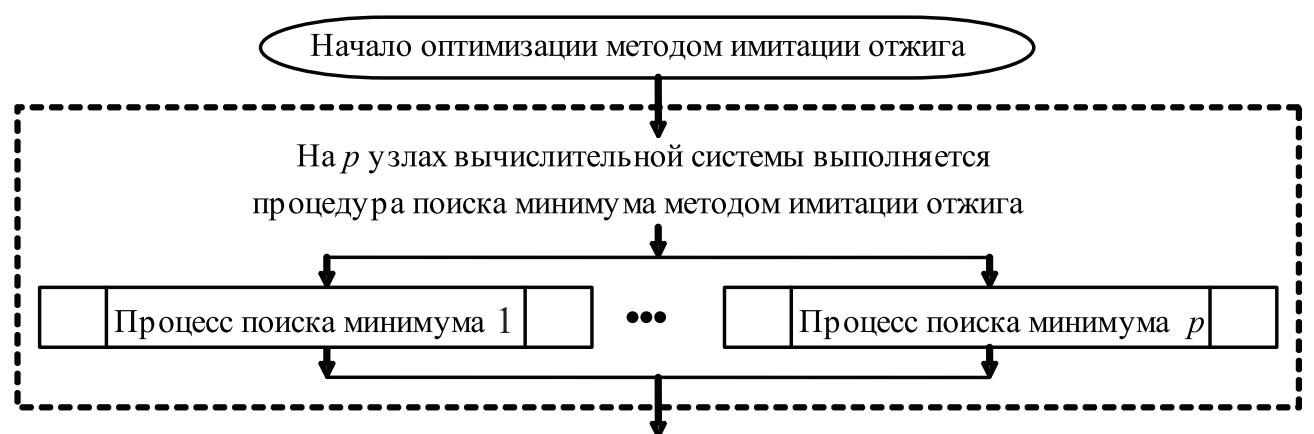

Выбор минимального значения ЦФ: $f\left(x_{i}\right)=\min \left(f\left(x_{1}\right), \ldots, f\left(x_{p}\right)\right)$

Выбор оптимальных параметров: $x_{\text {opt }}=x_{i}$

Завершение оптимизации методом имитации отжига

Рис. 1. Блок-схема алгоритма оптимизации методом имитации отжига, выполняемого на параллельной вычислительной системе. Пунктиром выделена часть алгоритма, выполняемая на клиентских машинах 


\section{2. Кластерный алгоритм}

Кластерный алгоритм является дальнейшим развитием алгоритма, который представлен выше. Основным отличием его является введение в выполняемую на каждом вычислительном узле параллельной системы последовательную часть алгоритма этапа обмена результатами между рабочими узлами.

Изначально на каждом из $p$ узлов параллельной вычислительной системы запускается алгоритм имитации отжига с разными начальными точками $x_{l}, 1 \leq l \leq p$. По выполнении заданного количества итераций рабочие узлы обмениваются текущими результатами, после чего продолжают работу, исходя из лучшего значения результата. После $t$ обменов рабочие узлы продолжают работу по получению индивидуального результата. По достижении условия завершения поиска главный узел вычислительной системы выбирает лучшее решение [2].

Таким образом, последовательная часть алгоритма имитации отжига по схеме больцмановского тушения приобретает соответствующий вид (рис. 2).

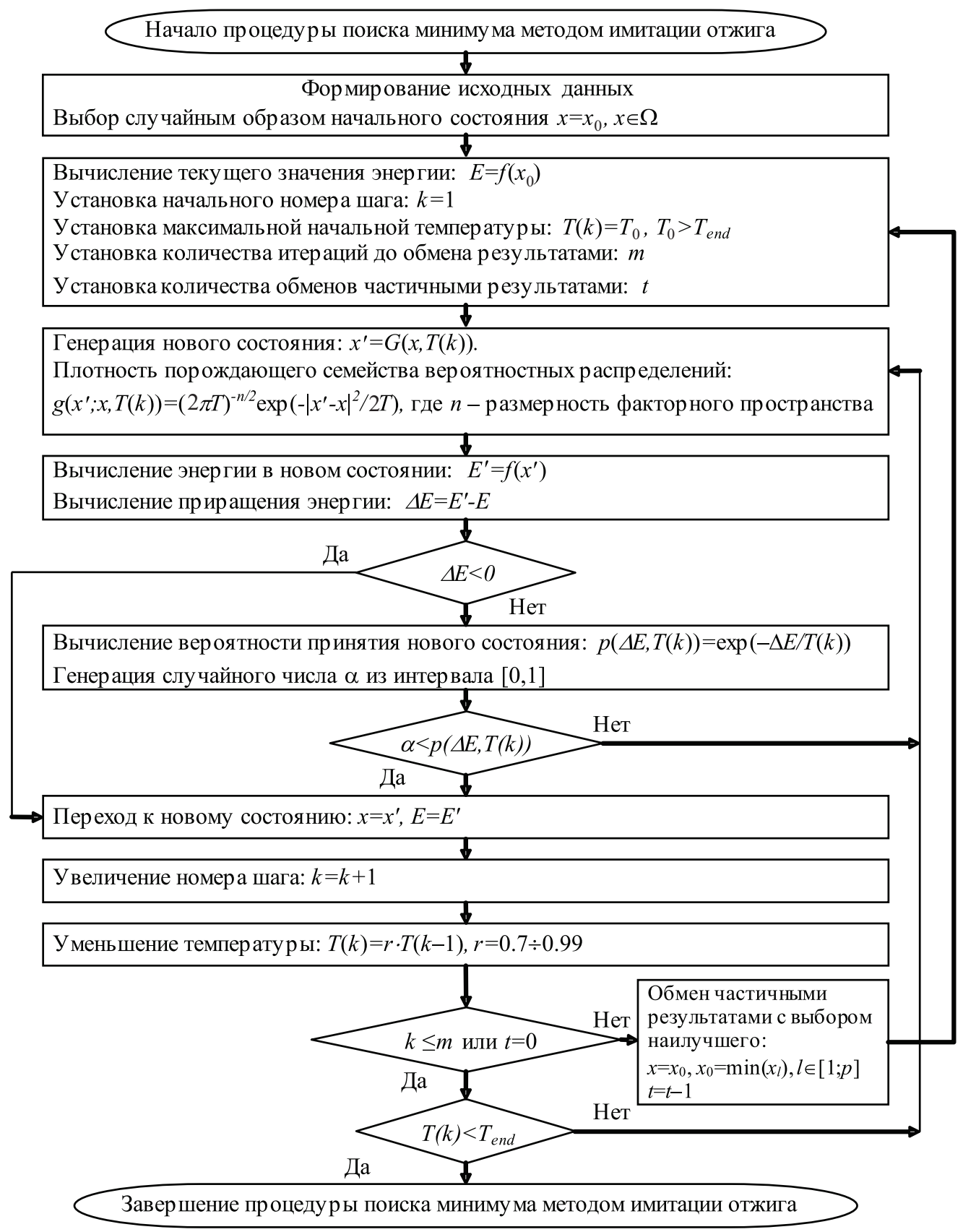

Рис. 2. Блок-схема последовательной части кластерного алгоритма имитации отжига по схеме больцмановского тушения с обменом результатами 


\section{3. Разбиение пространства решений на области}

В данном методе пространство решений алгоритма имитации отжига разбивается на несколько областей, в каждой из которых запускается последовательный алгоритм имитации отжига. В этом случае работа главного вычислительного узла, так же как и в упомянутых ранее адаптациях, сводится к выбору наилучшего решения среди полученных на рабочих узлах параллельной вычислительной системы.

Данный подход позволяет строить параллельные алгоритмы с низким трафиком обмена между параллельными процессами [2].

Построение алгоритма имитации отжига, основанного на этом подходе, требует решения следующих задач [3]:

1) разбиение исходного пространства корректных решений на несколько непересекающихся областей, дающих в объединении все пространство;

2) выбор начального корректного решения в каждой из областей;

3) введение операций преобразования решения таким образом, чтобы они были замкнуты в каждой из областей;

4) выбор способа распределения областей по узлам вычислительной системы и схемы отсечения «неперспективных» областей в ходе работы алгоритма.

Данный способ может эффективно применяться на широком классе задач. В то же время особенностью метода имитации отжига с разбиением на области является необходимость разработки способа разбиения для каждой решаемой задачи.

\section{3. ОПИСАНИЕ ЧИСЛЕННОГО ЭКСПЕРИМЕНТА И АНАЛИЗ ПОЛУЧЕННЫХ РЕЗУЛЬТАТОВ}

Оценка надёжности и эффективности распараллеленного алгоритма имитации отжига по схеме больцмановского тушения осуществлялась на многоэкстремальных ЦФ, с количеством параметров оптимизации $n$, равным 2 :

- гладкая многоэкстремальная функция:

$$
f\left(x_{1}, x_{2}\right)=-\frac{1}{\frac{x_{1}^{2}+x_{2}^{2}}{100}-\cos \left(x_{1}\right) \cos \left(\frac{x_{2}}{\sqrt{2}}\right)+2},
$$

где $-0.5<x_{j}<1, j=1,2, f_{\min }=-1$;

- многоэкстремальная функция Растригина:

$$
f\left(x_{1}, x_{2}\right)=20+x_{1}^{2}-10 \cos \left(2 \pi x_{1}\right)+x_{2}^{2}-10 \cos \left(2 \pi x_{2}\right),
$$

где $-0.5<x_{j}<1, j=1,2, f_{\min }=0$;

- негладкая 4 экстремальная функция:

$$
\begin{aligned}
& f_{1}=-5 e^{-3\left(\left|x_{1}+1\right|^{0.6}+\left|x_{2}+1\right|^{0.6}\right)}, \\
& f_{2}=-10 e^{-2\left(\left|x_{1}\right|+\left|x_{2}\right|\right)}, \\
& f_{3}=-7 e^{-2.5\left(\left|x_{1}-1\right|^{0.8}+\left|x_{2}-1\right|^{0.8}\right)}, \\
& f_{4}=-3 e^{-\left(\left|x_{1}-2\right|^{0.9}+\left|x_{2}-2\right|^{0.9}\right)}, \\
& f\left(x_{1}, x_{2}\right)=f_{1}+f_{2}+f_{3}+f_{4},
\end{aligned}
$$

где $-0.5<x_{j}<1, j=1,2, f_{\min }=-10.1$;

- негладкая 4 экстремальная функция, имеющая три равных по значению глобальных минимума:

$$
\begin{aligned}
& g_{1}=-7 e^{-3\left(\left|x_{1}+1\right|^{0.6}+\left|x_{2}+1\right|^{0.6}\right)}, \\
& g_{2}=-7 e^{-2\left(\left|x_{1}\right|+\left|x_{2}\right|\right)}, \\
& g_{3}=-7 e^{-2.5\left(\left|x_{1}-1\right|^{0.8}+\left|x_{2}-1\right|^{0.8}\right)}, \\
& g_{4}=-7 e^{-\left(\left|x_{1}-2\right|^{0.9}+\left|x_{2}-2\right|^{0.9}\right)}, \\
& f\left(x_{1}, x_{2}\right)=g_{1}+g_{2}+g_{3}+g_{4},
\end{aligned}
$$

где $-0.5<x_{j}<1, j=1,2, f_{\min }=-7.5$. 
Тестирование проводилось при следующих условиях: начальная температура $T_{0}=10^{9}$, конечная $T_{\text {end }}=0.1$, коэффициент изменения температуры $r=0.97$. При таких значениях параметров обеспечивалось оптимальное соотношение точности результата и времени вычисления алгоритма.

Результаты поиска минимума ЦФ (1), (2), (3) и (4) тремя распараллеленными методами имитации отжига в зависимости от числа одновременно работающих процессов $p$, усредненные по итогам 100 запусков вычислительной системы, приведены на рис. 3, a-2 для каждой из функций.
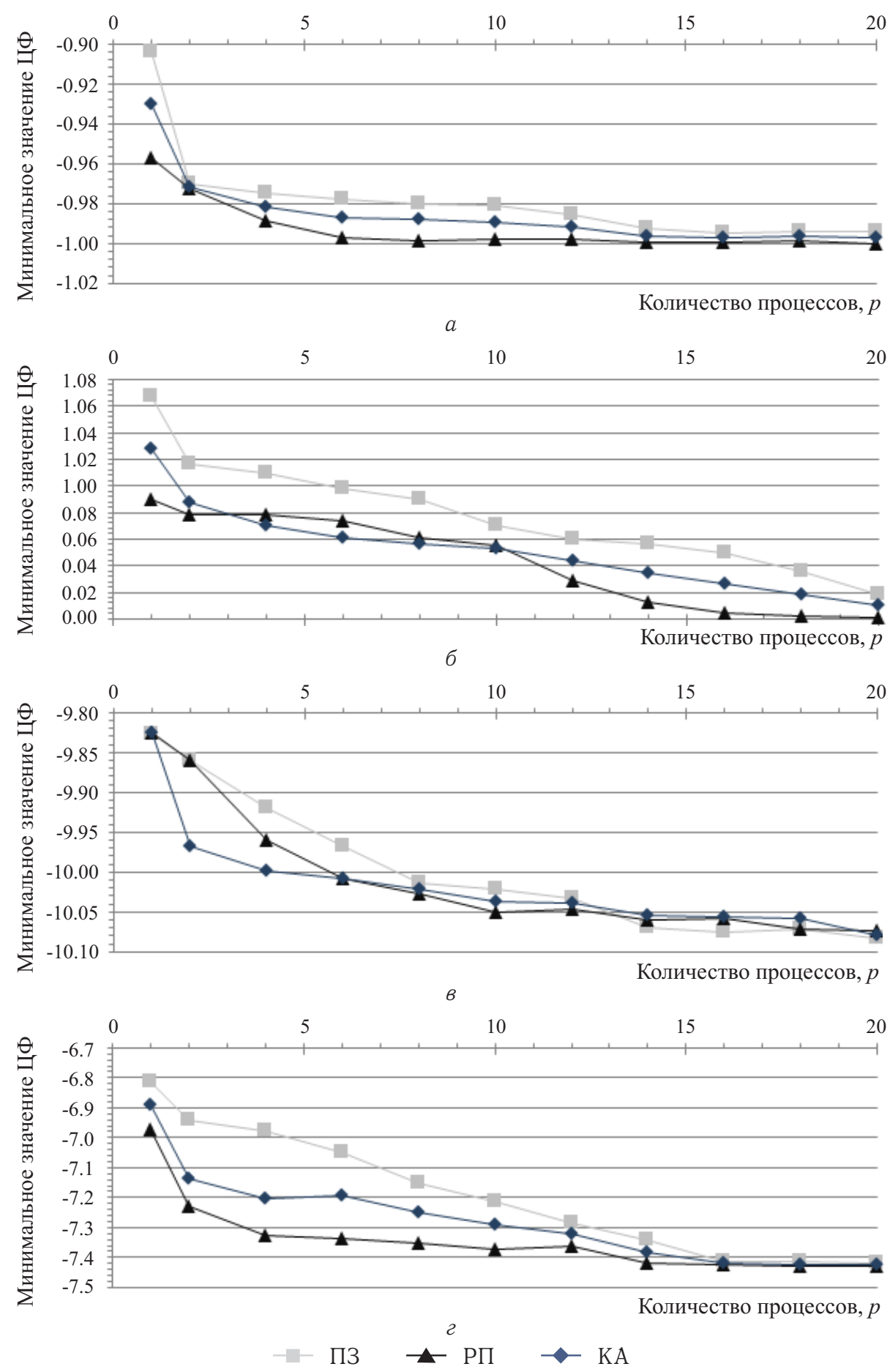

Рис. 3. Зависимость минимального значения целевой функции от количества одновременно работающих процессов при параллельном запуске (ПЗ), кластерном алгоритме (KА) и разбиении пространства решений на области (РП): $a-$ для гладкой многоэкстремальной функции, б - многоэкстремальной функции Растригина, в - для негладкой 4-экстремальной функции, г - для негладкой 4-экстремальной функции с тремя равными минимумами 
Как видно из рис. $3, a-2$, при малом количестве работающих процессов $p$ результат выдается с большим разбросом, но при увеличении $p$ результаты стабилизируются и приближаются к глобальному минимуму, так как поиск в этом случае ведётся в большей части заданной явными ограничениями области факторного пространства.

При этом увеличение числа параллельно работающих процессов $p$ с 1 до 20 приводит к уменьшению размеров области глобального минимума, оцениваемой по среднеквадратическому отклонению минимального значения ЦФ, на 60\%, 55\% и 50\% для разбиения пространства решений на области, кластерного алгоритма и параллельного запуска соответственно (рис. 4).

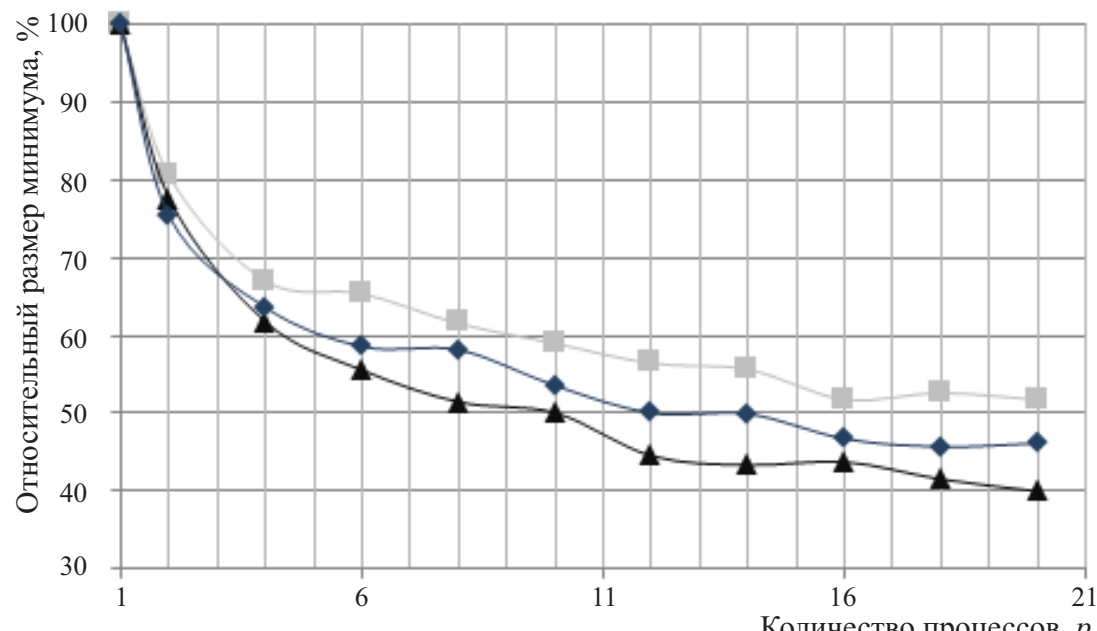

- Параллельный запуск алгоритма

Количество процессов, $p$

-

$\checkmark$ Кластерный алгоритм

Рис. 4. Зависимость размеров области глобального минимума от количества параметров оптимизации $n$ и числа $p$ одновременно работающих процессов поиска минимума методом имитации отжига по схеме больцмановского тушения

При решении реальных оптимизационных задач большое время занимает вычисление ЦФ. Соответственно эффективность метода оптимизации можно оценивать по количеству итераций алгоритма. Как видно из рис. 5, в случае параллельного запуска алгоритма на $p$ вычислительных узлах среднее число итераций алгоритма не будет зависеть от числа одновременно работающих процессов. Данная зависимость характерна и для кластерного алгоритма, так как количество итераций алгоритма задается пользователем и не зависит от числа работающих узлов. Однако с ростом числа узлов увеличивается время выполнения алгоритма. Это связано с задержкой в передаче и приеме данных, осуществляемых через сеть.

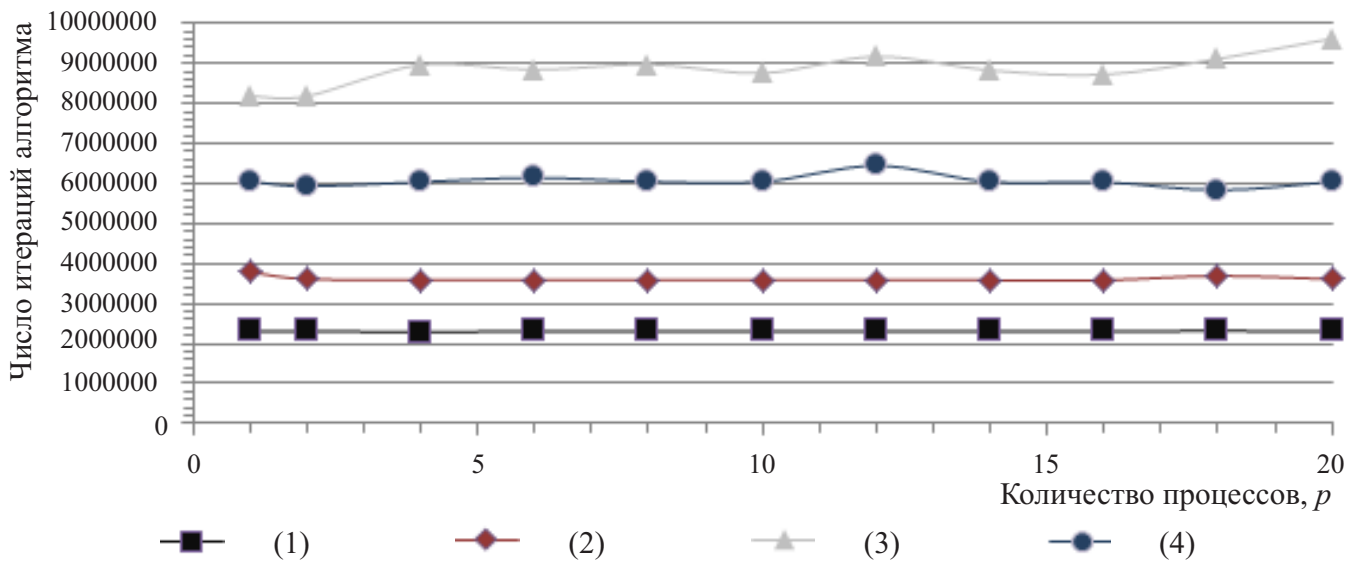

Рис. 5. Число итераций алгоритма поиска минимума ЦФ 1-4 методом параллельного запуска 
Число итераций сокращается с увеличением вычислительных узлов в случае разбиения пространства решений на области; соответствующая зависимость имеет экспоненциальный характер (рис. 6).

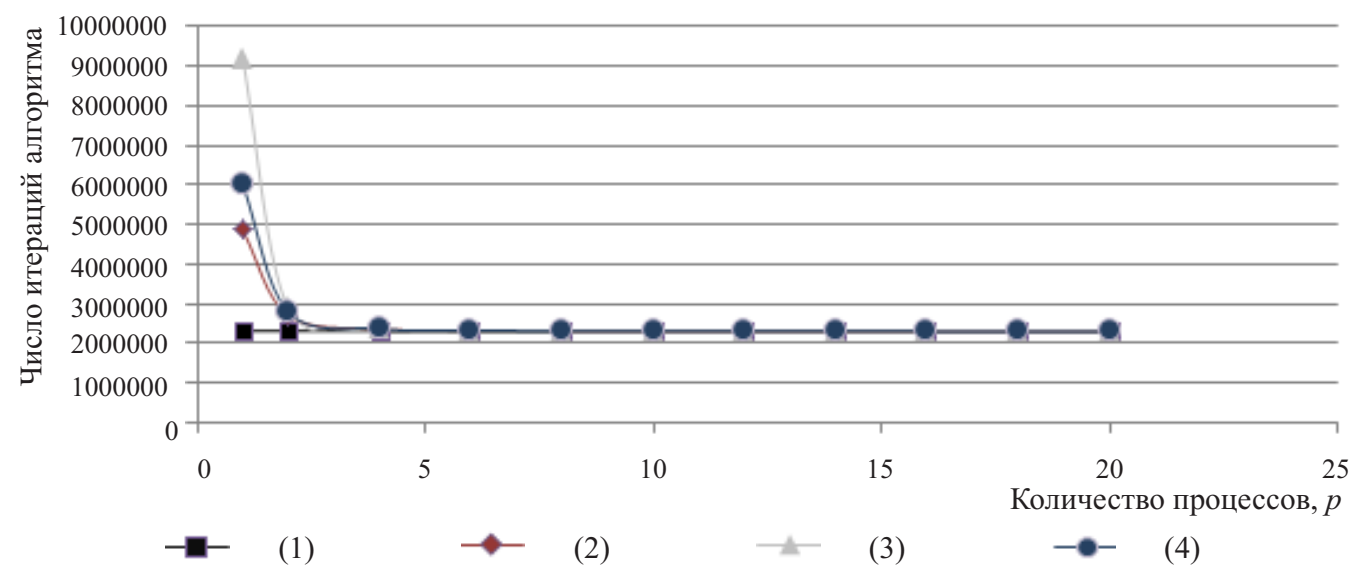

Рис. 6. Число итераций алгоритма поиска минимума ЦФ 1-4 методом разбиения пространства решений

Значительное влияние на количество вычислений ЦФ и соответственно на эффективность тестируемого метода оптимизации оказывает значение температурного коэффициента $r$. В ходе проведения численного эксперимента был сделан вывод, что изменение температурного коэффициента $r$ с 0.95 до 0.9999 приводит к увеличению времени вычислений ЦФ практически в 6 раз, однако при этом точность нахождения решения повышается.

\section{ЗАКЛЮЧЕНИЕ}

В ходе проведенной работы были рассмотрены и реализованы 3 варианта распараллеливания алгоритма оптимизации многоэкстремальной целевой функции двух переменных с явными ограничениями, основанного на стохастическом методе имитации отжига, и проведен анализ эффективности работы каждого из способов распараллеливания.

\section{Библиографический список}

1. Лопатин А. С. Метод отжига // Стохастическая оптимизация в информатике : межвуз. сб. СПб. : Изд-во СПбГУ, 2005. Вып. 1. С. 133-149.

2. Савин А. Н, Тимофеева Н. Е. Применение алгоритма оптимизации методом имитации отжига на системах параллельных и распределённых вычислений // Изв.
Сарат. ун-та. Нов. сер. Сер. Математика. Механика. Информатика. 2012. Т. 12, вып. 1. С. 110-116.

3. Kirkpatrick S. A., Gelatt C. D., Vecchi M. P. Optimization by simulated annealing // Science. N.S. 1983. Vol. 220, № 4598. P. 671-680.

\section{The Effectiveness Analysis of Several Parallel Algorithms Based on Simulated Annealing Method of Global Optimization Problem Solving}

\section{A. V. Vysotsky, A. S. Tarakanov, K. I. Sholomov, N. E. Timofeeva, A. A. Eroftiev}

Saratov State University, Russia, 410012, Saratov, Astrahanskaya st., 83, alex_v2008@mail.ru, tarakanov.alexey@gmail.com, sholomov@mail.ru, timofeevane@info.sgu.ru, eroftiev.andrey@gmail.com

This article presents the results of the development of a parallel computing system and testing its capabilities applied to solving scientific and educational problems. Three parallel variants of the simulated annealing algorithm are proposed and implemented for multiextreme criterion function of two variables with explicit constraints. The reliability and performance of parallel versions of the algorithm, depending on their parameters and the number of working nodes in parallel computing system, is investigated. It is shown that proposed parallel variants of simulating annealing algorithm allow successful finding the global minimum of multiextreme criterion function.

Key words: global optimization, simulated annealing, multiextreme criterion function, explicit constraints, parallel computing. 


\title{
References
}

1. Lopatin A. S. Simulated Annealing. Stokhasticheskaia optimizatsiia v informatike: mezhouz. sb. [Stochastic Optimization in Informatics]. St. Petersburg, 2005, iss. 1, pp. 133-149 (in Russian).

2. Savin A. N, Timofeeva N. E. Using Optimization Algorithm Based on Simulated Annealing on Parallel and

Distributed Computing Systems. Izv. Sarat. Univ., N.S., Ser. Math. Mech. Inform., 2012, vol. 12, iss. 1, pp. 110116 (in Russian).

3. Kirkpatrick S. A., Gelatt C. D., Vecchi M. P. Optimization by simulated annealing. Science, N.S., 1983, vol. 220 , no. 4598 , pp. 671-680.

\section{ОБ ОШИБКЕ ПРИБЛИЖЕНИЯ ДЕРЕВЬЯМИ СЦЕНАРИЕВ ЕДИНИЧНОЙ ГЛУБИНЫ}

\begin{abstract}
Е. А. Захарова ${ }^{1}$, С. П. Сидоров ${ }^{2}$
${ }^{1}$ Аспирант касредры математической экономики, Саратовский государственный университет им. Н. Г. Чернышевского, zakharova_e@yahoo.com

${ }^{2}$ Кандидат фризико-математических наук, доцент кафредры математической экономики, Саратовский государственный университет им. Н. Г. Чернышевского, sidorovsp@info.sgu.ru

Обозначим через $\Lambda_{n}$ множество всех деревьев сценариев глубины 1 с числом сценариев $n$ на $[0,1]$. Пусть $X=(0 \leq$ $\left.\leq x_{1}<\ldots<x_{n} \leq 1\right)$ и обозначим $\Lambda_{n}(X)$ множество всех деревьев сценариев глубиной 1 с $n$ сценариями $X=\left(0 \leq x_{1}<\ldots<x_{n} \leq 1\right)$. Пусть $G$ есть вероятностное распределение, определенное на $[0,1]$, и $H$ - некоторый класс измеримых на $[0,1]$ функций. Положим $d_{H, X}(G)=\inf _{\tilde{G} \in \Lambda_{n}(X)} d_{H}(G, \tilde{G})$ и $d_{H}(G)=\inf _{\tilde{G} \in \Lambda_{n}} d_{H}(G, \tilde{G})$, где $d_{H}(G, \tilde{G}):=\sup _{h \in H}\left|\int h d G-\int h d \tilde{G}\right|$. Цель работы состоит в нахождении величин $d_{H}(G, X)$ и $d_{H}(G)$ для случая, когда множество $H$ есть подмножество всех алгебраических многочленов степени не выше $n$. Таким образом, мы рассматриваем задачу приближения меры $G$ деревом сценариев в смысле равенства первых $n$ моментов.
\end{abstract}

Ключевые слова: деревья сценариев, метод моментов.

\section{ВВЕДЕНИЕ}

В задачах портфельного инвестирования и управления риском используются методы стохастического программирования, при этом для генерации многомерных случайных величин, соответствующих реальным процессам (поведение на рынке ценных бумаг, управление коммунальными услугами, цены на топливо или электричество, доставка товаров и т.д.), используется определенный набор сценариев и соответствующих им вероятностей $[1,2]$. В большинстве случаев количество таких сценариев слишком велико и их необходимо аппроксимировать некоторым набором с меньшим количеством сценариев, при этом возникает определенная погрешность. При использовании деревьев сценариев задается начальное количество сценариев и соответствующих им вероятностей выполнения этих сценариев. Данная проблема получила наибольшее развитие в начале 2000 годов [3]. Вслед за финансовым кризисом 1998 года появилась необходимость точнее просчитывать риски, в том числе и для сценариев с малой вероятностью, но увеличение количества допустимых сценариев влечет за собой усложнение моделей и трудности в ее компьютерном моделировании. Использование же деревьев сценариев дает возможность упростить модель.

В работе [4] особая важность придается алгоритмам генерации случайных чисел, рассматриваются случаи генерации достаточного количества случайных чисел, соответствующим параметрическим и непараметрическим стохастическим моделям. В статье [5] описывается метод, основанный на использовании нелинейного программирования. Главной идеей этого метода является сокращение разницы между статистическими свойствами случайных величин, что дает больший контроль над распределением.

В настоящей работе оценивается ошибка приближения произвольного непрерывного распределения деревьями сценариев единичной глубины, имеющих равные моменты фиксированных порядков.

Пусть $G$ и $\tilde{G}$ есть два вероятностных распределения, определенных на $[0,1]$. Пусть $H-$ некоторый класс измеримых на $[0,1]$ функций.

Определим $d_{H}(G, \tilde{G})$ следующим образом:

$$
d_{H}(G, \tilde{G})=\sup _{h \in H}\left|\int h d G-\int h d \tilde{G}\right| .
$$

